\title{
Topical Management of Acute Radiation Dermatitis in Breast Cancer Patients: A Systematic Review and Meta-Analysis
}

\author{
FATIMAH HARUNA, ANDREA LIPSETT and LAURE MARIGNOL \\ Applied Radiation Therapy Trinity (ARTT), Discipline of Radiation Therapy, \\ School of Medicine, Trinity College Dublin, Dublin, Ireland
}

\begin{abstract}
Aim: To evaluate the efficacy of topical corticosteroids in managing acute radiation dermatitis $(R D)$ in female breast cancer patients. Materials and Methods: MEDLINE, EMBASE, CINAHL, CENTRAL, ScienceDirect, Google Scholar and Clinicaltrials.gov. were searched up to and including March 2017 to identify Randomised Controlled Trials (RCTs) assessing topical corticosteroids for the management and prevention of acute RD. Results: Ten RCTs (919 participants) were identified. Meta-analysis, including results for 845 participants, demonstrated significant benefits of topical corticosteroids in preventing the incidence of wet desquamation (OR: 0.29; 95\%CI: 0.19-0.45; $p<0.0001$ ) and reducing the mean RD score (SMD: $-0.47,95 \% C I:-0.61--0.33, p<0.00001$ ). Conclusion: Topical corticosteroids impacted on the incidence of wet desquamation and the average RD score observed in female breast cancer patients. The use of topical corticosteroids can reduce pruritus in participants and improve quality of life.
\end{abstract}

Radiation induces damage to the skin, activating inflammatory pathways and causing cytokines overproduction $(1,2)$. Radiation dermatitis is a common acute side-effect that occurs within hours to weeks after the start of radiotherapy $(3,4)$ and affects more than $87 \%$ of patients $(2,5)$. Radiation dermatitis can limit the therapeutic dose delivered to patients and can, sometimes, lead to a break in treatment, thus potentially compromising local control and survival outcome $(1,6)$. It can also have a considerable impact on patients' quality of life $(7$,

This article is freely accessible online.

Correspondence to: Laure Marignol, Applied Radiation Therapy Trinity (ARTT), Discipline of Radiation Therapy, School of Medicine, Trinity College Dublin, Ireland. E-mail: marignol@tcd.ie

Key Words: Radiation dermatitis, breast cancer, corticosteroids, wet desquamation, review.
8). Despite significant development in radiotherapy techniques, efficacious interventions in the prevention of acute skin reactions is still lacking and current evidence is unable to provide adequate guidelines for the management of this sideeffect $(9,10)$. Studies have examined numerous topical agents such as Aloe Vera, aqueous cream, Calendula, petrolatum and sulcrafate cream as a means to reducing the dehydrating effects of radiation dermatitis $(6,11,12)$. However, the results in terms of managing radiation dermatitis and treatment related pain have not been clinically significant $(13,14)$. Corticosteroids have anti-inflammatory properties and are known to down-regulate cytokine gene expression, making them ideal for managing radiation dermatitis (15-17). A systematic review by Bolderston et al. concluded that there is limited evidence to support or oppose the use of topical agents for the management of acute radiation dermatitis (18).

Skin care advice given to breast cancer patients varies among institutions (19). No gold-standard for the appropriate management of this side-effect exists and in most cases the decision of care is left at the discretion of the health care professional rather than clinical evidence $(20,21)$. Clinical advice is often given based on anecdotal evidence (22).The present systematic review and meta-analysis was conducted to assess the potential efficacy of corticosteroids in the management of RD and its impact on pain and quality of life in female breast cancer patients. However, the anticipated adverse effect associated with steroids is not evaluated and is beyond the scope of this review. We restricted our review to one site only due to radiotherapy dose variations used in different sites.

\section{Materials and Methods}

Search strategy for identification of studies. The electronic literature search was conducted up to and including March 2017. The search terms used were corticosteroid, radiation dermatitis, breast cancer, management, and prevention. The reference lists of potentially eligible studies were subsequently hand searched to identify 
Table I. Search strategy for identification of studies.

\section{EMBASE}

Emtree: breast cancer: 373,140 records, radiation dermatitis: 3,131 records, steroid: 1,363,423 records

Query Builder: "breast cancer"/exp and "radiation dermatitis"/exp and "steroid"/exp: 107 items including 7 of the trials

\section{MEDLINE}

Search Builder: ("Radiodermatitis"[Mesh] and "Breast Neoplasms"[Mesh]) and "Steroids"[Mesh]: 18 items including 8 of the trials

ScienceDirect

Advanced search:

Search for: radiation dermatitis (TITLE-ABSTR-KEY) and breast cancer (TITLE-ABSTR-KEY)

Refine your search: Journals; Years: 2000-present: 78 items including 4 of the trials

Google Scholar

Radiation dermatitis (with all words) breast cancer (with the exact phrase) in the title of the article, Return articles dated between 2000-2017:

78 items including 2 of the trials

CINAHL

Search 1: (MH "Breast Neoplasms+") 59,993 records, Search 2: "radiation dermatitis" 108 records, Search 3: (MH "Steroids+") 52,686 records

Boolean Operator S1 and S2 and S3: 5 items including 2 of the trials

CENTRAL

MeSH descriptor: [Radiodermatitis] explode all trees 129 records, MeSH descriptor: [Breast Neoplasms] explode all trees 10104 records,

MeSH descriptor: [Steroids] explode all trees 44,093 records

"radiodermatitis" and "breast neoplasms" and "steroids": 4 records including 2 of the trials

Clinicaltrials.gov

"Breast cancer" and "radiation dermatitis": 38 records: 4 completed trials with results including 1 of the trials

Records identified through database searching: $107+18+78+78+5+4+4=294$

Table II. Methodological quality summary: Jadad et al. scale for reporting randomised controlled trials.

\begin{tabular}{|c|c|c|c|c|c|c|c|c|c|c|}
\hline $\begin{array}{l}\text { Jadad's Methodo- } \\
\text { logical quality item }\end{array}$ & $\begin{array}{l}\text { Bostrom } \\
\text { et al. } \\
(2001)\end{array}$ & $\begin{array}{l}\text { Schmuth } \\
\text { et al. } \\
(2002)\end{array}$ & $\begin{array}{l}\text { Farhan } \\
\text { et al. } \\
\text { (2003) }\end{array}$ & $\begin{array}{l}\text { Shukla } \\
\text { et al. } \\
(2006)\end{array}$ & $\begin{array}{l}\text { Omidvari } \\
\text { et al. } \\
(2007)\end{array}$ & $\begin{array}{l}\text { Miller } \\
\text { et al. } \\
(2011)\end{array}$ & $\begin{array}{l}\text { Ulff } \\
\text { et al. } \\
\text { (2013) }\end{array}$ & $\begin{array}{l}\text { Hindley } \\
\text { et al. } \\
(2014)\end{array}$ & $\begin{array}{l}\text { Meghrajani } \\
\text { et al. } \\
(2016)\end{array}$ & $\begin{array}{l}\text { Ulff } \\
\text { et al. } \\
(2017)\end{array}$ \\
\hline Randomised & Yes & Yes & Yes & Yes & Yes & Yes & Yes & Yes & Yes & Yes \\
\hline $\begin{array}{l}\text { Method of } \\
\text { randomisation } \\
\text { is appropriate }\end{array}$ & Yes & Yes & No & No & Yes & Yes & No & Yes & Yes & Yes \\
\hline Blinding & Yes & Yes & Yes & N/A & Yes & Yes & Yes & Yes & Yes & Yes \\
\hline $\begin{array}{l}\text { Method of } \\
\text { blinding is } \\
\text { appropriate }\end{array}$ & Yes & Yes & Yes & N/A & Yes & Yes & Yes & No & Yes & Yes \\
\hline An account of all patient & Yes & Yes & Yes & Yes & Yes & Yes & Yes & Yes & Yes & Yes \\
\hline Total Jadad score & $5 / 5$ & $5 / 5$ & $4 / 5$ & $2 / 3$ & $5 / 5$ & $5 / 5$ & $4 / 5$ & $4 / 5$ & $5 / 5$ & $5 / 5$ \\
\hline
\end{tabular}

additional studies. A search strategy was developed (Table I) and a PRISMA Flow chart was used in the study selection process (Figure 1) (23). RCTs assessing management and/or prevention of radiation dermatitis were included.

Types of participants and interventions. Eligible trials included female breast cancer patients of any stage receiving minimum adjuvant EBRT dose of 40 Gy to the breast or chest wall. RCTs were also included if participants in addition to breast/ chest wall irradiation had treatment to regional lymph nodes. Studies were included if the intervention was aimed at preventing or managing $\mathrm{RD}$ in participants. Topical corticosteroid must have been used solely for or as part of the intervention with the intent of preventing or managing RD. The comparison could be placebo, no treatment, or a topical non-steroidal agent. Studies comparing only topical steroids or where steroids were not used as intervention were 
excluded. Trials must have reported the method of measuring RD and must statistically assess it as a primary outcome.

Types of outcomes. Eligible RCTs reported severity of acute RD in both arms of studies. Incidence of wet desquamation was assessed as a primary outcome. Other outcomes evaluated were mean RD score, pain, pruritus, burning sensation and Qol.

Methodological quality. The internal quality of the included studies was assessed using the Jadad et al. scale for reporting randomised controlled trials (Table II) (24), and were assigned a quality score between 0 (low) and 5 (high). The Cochrane Collaboration's tool for assessing risk of bias was also employed (25) (Figure 2). Funnel plots were constructed to assess for the possibilities of publication bias and heterogeneity in the studies.

Statistical analysis. Two independent collectors extracted the data. Data on participants, intervention and outcome were extracted and these included beam energy, surgery type, overall RT dose, steroid application (Table III).

Statistical Power was calculated in RCTs using a two-sided test of significance $(8,26,27)$, test was not reported for in three RCTs $(28,29)$ and no statistical power calculation was present for four $(7$, 30-32). Descriptive statistics were reported in all studies for age (quantitative numerical data) including mean, median, and range (7, $8,26-33)$. Other trials reported mean and range field size $(29,33)$, field area (33), range of total radiation dose $(7,26)$, median number of nodes and range (8).

In one study homogeneity of study arms was controlled using non-parametrical tests, Kruskal-Wallis test and Friedman test (33). The factors used were age, radiotherapy field size and field arrangement and type and number of chemotherapy courses prescribed before radiotherapy. The method of ensuring homogeneity was unclear in other RCTs however the authors stated that the features of the two groups were comparable and balanced (7, 8, 26-32).

Kruskal-Wallis test $(7,32)$, Chi-square tests $(31,33)$ and unpaired $t$-tests (7) were employed to assess the differences in RD scores between study arms. The mean maximum score was assessed using a single two-sample $t$-test (26). The differences in mean responses of patients reported symptoms and Qol were analysed using the Kruskal-Wallis test. The intention-to-treat principle and two tailed tests were used in two studies $(8,29)$. In one of the two studies the Mann-Whitney $U$-test was used for the statistical analyses of RD and patients reported symptoms (8). In scoring RD observed in participants, a modified RTOG (27), a standard RTOG score $(28,31-33)$ and CTCAE version 3.0 was used $(26,29)$. Clinical scales were used in 3 RCTs $(7,8,30)$.

The Visual Analogue Scale (VAS) and the Skindex-16 questionnaire were used to measure subjective symptoms in six RCTs $(7,8,26,28,29,32)$. The Dermatology Life Questionnaire Index (DLQI) was used to measure Qol in three (27-29). Pain was reported using the terms mild, moderate and severe (31). Due to the heterogeneity in the reporting of symptoms and Qol in the studies eliminating the possibilities of pooling, the data was summarised.

Forest Plots were generated wherever possible for the incidence of wet desquamation and RD scores. As mentioned previously data for symptoms and Qol were too heterogeneous to include in a forest plot and no threshold were required. The threshold for acute RD was the onset of wet desquamation or grade 3 CTCAE/RTOG scale.

\section{PRISMA Flow Diagram}

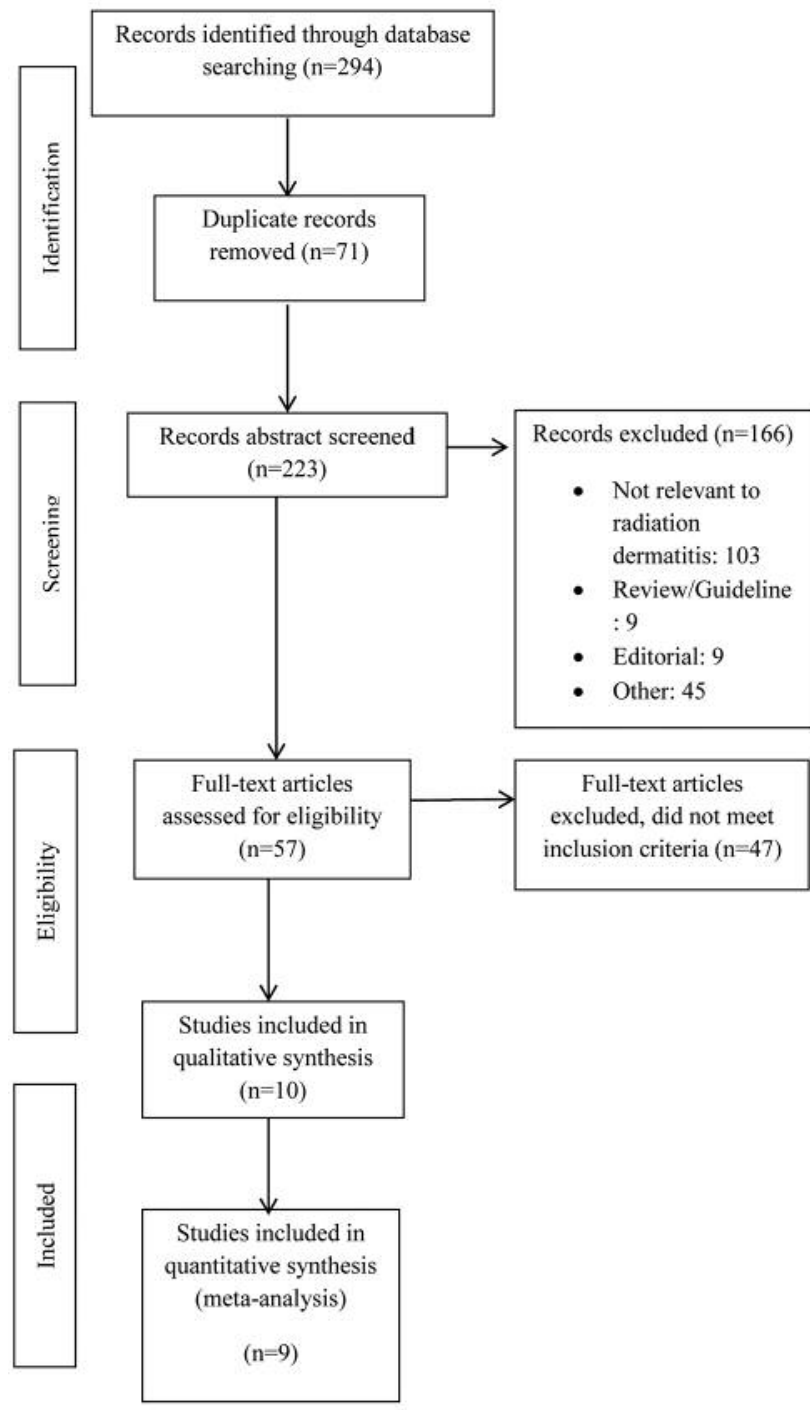

Figure 1. PRISMA flow diagram for identification of included studies.

This was the endpoint of this review. All forest and funnel plots were constructed using The Cochrane Collaboration's Review Manager, version 5.3.

\section{Results}

Description of studies. Ten studies meeting the inclusion criteria for this review were published between 2001 and 2017 (7, 8, 26-33). The main characteristics of the included studies are presented in Table III. In the ten included studies, 919 participants were randomly assigned to steroid versus nonsteroidal agent except for one study where the control arm had no treatment (30). The steroids assessed were mometasone 


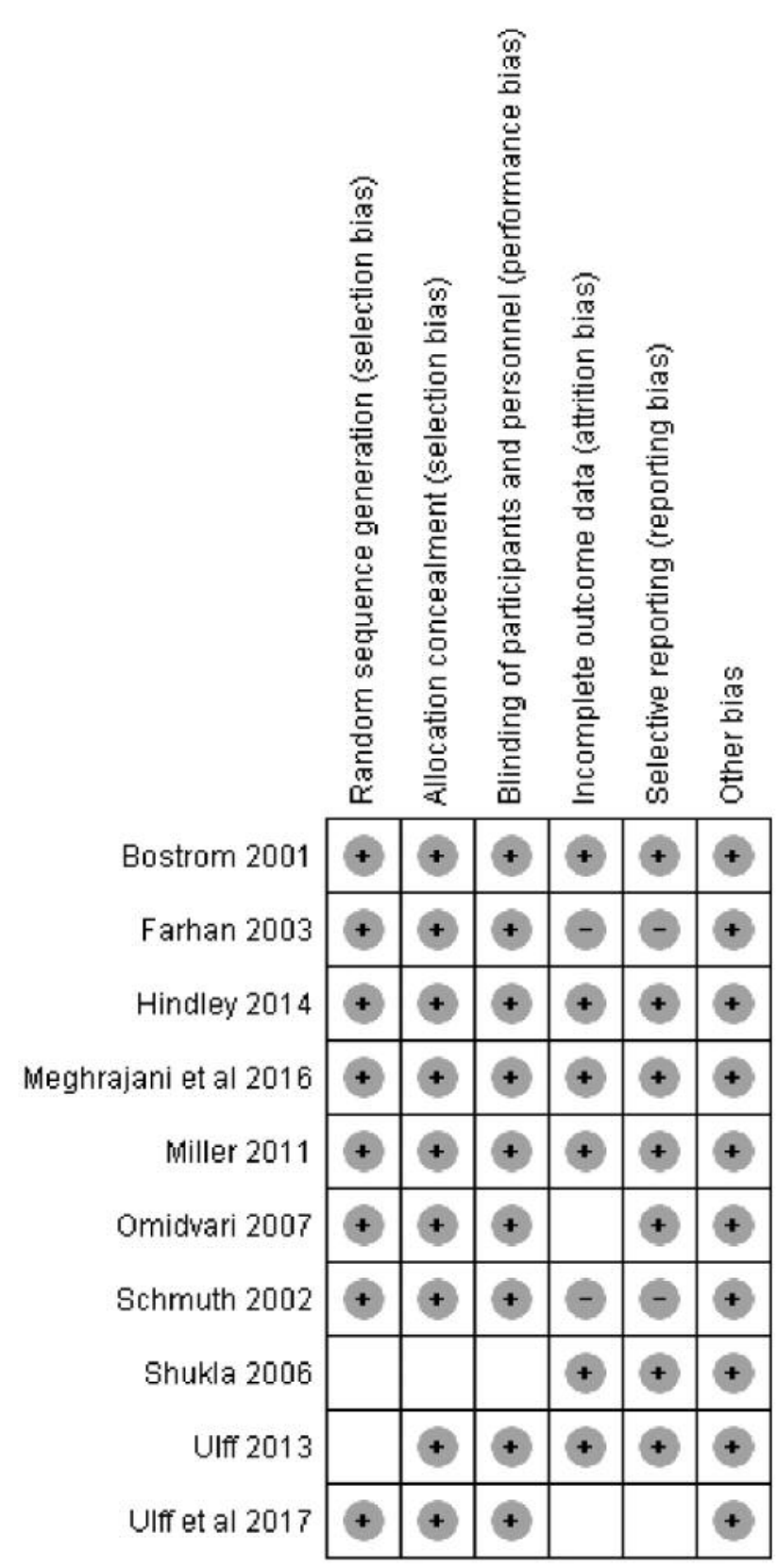

Key

Low risk of bias:

Unclear risk of bias:

High risk of bias:

Figure 2. Risk of bias summary: review author's judgements about each risk of bias item for each included study. furoate $(8,26,27)$, beclomethasone(30), methyl predinisolone (7), betamethasone (28, 31-33) and hydrocortisone (29). All the participants had breast cancer and underwent either mastectomy or breast conservative therapy. The type of surgery was comparable within the trials (Table III). Participants were females with age ranging from 27-97 years. Radiotherapy dose ranged from 40 Gy - >56 Gy. Treatment were applied once/twice daily to the breast or chest wall from the first fraction of radiotherapy to the last fraction and in some studies up to 3 weeks after radiotherapy. The frequency of treatment application differs between the trials (Table III). Participants were not allowed to apply any other medication to the breast or chest wall during the study duration.

Risk of bias in included studies. The results of the Jadad et al. Scale assessed by two independent observers for the individual studies have been included (Table II). From the Jadad et al. scale (5 questions), all of the trials scored at least 4 out of 5 except one study (30). In this study blinding could not be done. It was scored 2 out of a maximum of 3 points. According to the Jadad et al. scale all studies would be deemed to be moderate to high quality. In accordance with the Cochrane risk of bias tool (25) (Figure 2), four of the studies had low risk of bias $(8,26,27,29)$, two had high risk features $(7,31)$ and four had unclear risk in one or more domains $(28,30,32,33)$. The intention-to-treat approached was employed by Bostrom et al. and Meghrajani et al. thereby rated low risk for incomplete outcome data and selective reporting $(8,29)$. Drop out was highest in Schmuth et al. with $8.7 \%$ (7) (Table III). Two participants dropped out of this study compared to ten in Miller et al. however due to its small sample size, it had the highest percentage of drop outs relative to its sample size. Overall the methodological qualities of all trials were reasonable.

Incidence of wet desquamation. Nine of the ten RCTs stated the incidence of wet desquamation in their results, accounting for 845 patients out of 919 (8, 26-33). Eight out of the nine studies showed a lower incidence of wet desquamation in the steroid arm versus the control $(8,27-33)$. Pooling the results for nine RCTs, identified that topical corticosteroid significantly reduce the incidence of wet desquamation, (OR: $0.29 ; 95 \% \mathrm{CI}=0.19-0.45 ; p<0.0001)$. One RCT was excluded from this analysis because incidence of wet desquamation was not presented (7). There was no evidence of heterogeneity in the pooled data following both quantitative $\left(\mathrm{Chi}^{2}=3.85 ; \mathrm{df}=8\right.$; $p=0.87 ; \mathrm{I}^{2}=0 \%$ ) (Figure $3 \mathrm{~A}$ ) and qualitative analyses (Figure 3B). Test of overall effect showed that, the risk of developing wet desquamation is approximately 5 times less likely with the use of topical corticosteroids (Figure 3A).

Mean radiation dermatitis score. Mean $\mathrm{RD}$ scores were reported in nine studies. Mean RD scores and standard 
Table III. Characteristics of included studies.

\begin{tabular}{|c|c|c|c|c|c|c|c|c|c|c|}
\hline & $\begin{array}{l}\text { Bostrom } \\
\text { et al. } \\
(2001)\end{array}$ & $\begin{array}{l}\text { Schmuth } \\
\text { et al. } \\
\text { (2002) }\end{array}$ & $\begin{array}{l}\text { Farhan } \\
\text { et al. } \\
(2003)\end{array}$ & $\begin{array}{l}\text { Shukla } \\
\text { et al. } \\
(2006)\end{array}$ & $\begin{array}{l}\text { Omidvari } \\
\text { et al. } \\
(2007)\end{array}$ & $\begin{array}{l}\text { Miller } \\
\text { et al. } \\
(2011)\end{array}$ & $\begin{array}{l}\text { Ulff } \\
\text { et al. } \\
\text { (2013) }\end{array}$ & $\begin{array}{l}\text { Hindley } \\
\text { et al. } \\
(2014)\end{array}$ & $\begin{array}{l}\text { Meghrajani } \\
\text { et al. } \\
(2016)\end{array}$ & $\begin{array}{l}\text { Ulff } \\
\text { et al. } \\
(2017)\end{array}$ \\
\hline \multicolumn{11}{|l|}{ Participants } \\
\hline Sample size (n) & $\begin{array}{c}50(25 \\
\mathrm{MF} / 25 \\
\text { placebo })\end{array}$ & $\begin{array}{l}23(12 \\
\text { methyl/ } \\
11 \mathrm{dex})\end{array}$ & $\begin{array}{c}76(38 \\
\text { beta/38 } \\
\text { placebo) }\end{array}$ & $\begin{array}{c}60(30 \\
\text { beclo/30 } \\
\text { no trt })\end{array}$ & 58 & $\begin{array}{c}176(90 \\
\text { MF/86 } \\
\text { placebo) }\end{array}$ & $\begin{array}{c}104(53 \\
\text { beta/24 essex/ } \\
27 \text { cano }\end{array}$ & $\begin{array}{l}120(62 \\
\mathrm{MF} / 58 \\
\mathrm{D})\end{array}$ & $\begin{array}{c}50(23 \\
\text { hydro/ } \\
27 \text { placebo })\end{array}$ & $\begin{array}{c}202(102 \\
\text { beta/100 } \\
\text { essex })\end{array}$ \\
\hline $\begin{array}{l}\text { Age range/ } \\
\text { mean }+/-\mathrm{SD}\end{array}$ & $47-76$ & $35-75$ & $27-70$ & $28-60$ & $34-66$ & $27-89$ & $28-90$ & $\begin{array}{c}59+/-11 \mathrm{MF} / \\
60+/-10 \mathrm{D}\end{array}$ & $31-70$ & $27-97$ \\
\hline surgery & BCS & $\begin{array}{l}\text { BCS \& } \\
\text { MRM }\end{array}$ & $\begin{array}{l}\text { BCS \& } \\
\text { MRM }\end{array}$ & $\begin{array}{l}\text { BCS \& } \\
\text { MRM }\end{array}$ & MRM & $\begin{array}{l}\text { BCS \& } \\
\text { MRM }\end{array}$ & $\begin{array}{l}\text { BCS \& } \\
\text { MRM }\end{array}$ & $\begin{array}{c}\text { BCS \& } \\
\text { MRM (+bolus) }\end{array}$ & MRM & $\begin{array}{l}\text { BCS \& } \\
\text { MRM }\end{array}$ \\
\hline RT machine & Linac & $\begin{array}{l}\text { Linac }+/- \\
\text { electron } \\
\text { boost }\end{array}$ & Cobalt-60 & Cobalt-60 & $\begin{array}{c}\text { Superficial } \\
\text { X-rays }\end{array}$ & - & Linac & $\begin{array}{l}\text { Linac +/- } \\
\text { electron } \\
\text { boost }\end{array}$ & Cobalt- 60 & Linac \\
\hline Beam energy & $\begin{array}{l}5 \mathrm{MV} \\
\text { photons } \\
\end{array}$ & $\begin{array}{c}8 \mathrm{MV} \\
\text { photons } \\
+/-14 \mathrm{MeV} \\
\text { electrons }\end{array}$ & - & - & - & - & - & 6-10 MV & - & - \\
\hline $\begin{array}{l}\text { RT total dose } \\
\text { (Gy) }\end{array}$ & $\begin{array}{l}54-56 \mathrm{~Gy} \\
(2 \mathrm{~Gy} / \#) \mathrm{G}\end{array}$ & $\begin{array}{c}56+/-4 \\
\text { Gy }(2 \mathrm{~Gy} / \#)\end{array}$ & $\begin{array}{c}50 \mathrm{~Gy} \\
{[\mathrm{MRM}]-} \\
60 \mathrm{~Gy} \\
{[\mathrm{BCS}]} \\
(2 \mathrm{~Gy} / \#)\end{array}$ & $\begin{array}{c}50+/- \\
16 \mathrm{~Gy} \\
{[\text { boost] }} \\
2 \mathrm{~Gy} / \#\end{array}$ & $\begin{array}{c}50 \mathrm{~Gy} \\
(2 \mathrm{~Gy} / \#)\end{array}$ & $\begin{array}{l}>50(1.75- \\
2.12 \mathrm{~Gy} / \#)\end{array}$ & $\begin{array}{l}50(2 \\
\text { Gy/\#) }\end{array}$ & $\begin{array}{c}40(2.67 \\
\mathrm{Gy} / \#)+/- \\
10(2 \mathrm{~Gy} / \#)\end{array}$ & $\begin{array}{c}50+/- \\
10 \mathrm{~Gy}[\text { boost] } \\
2 \mathrm{~Gy} / \#\end{array}$ & $\begin{array}{c}42.56 / 50 \\
\text { Gy }(2.66 / \\
2 \mathrm{~Gy} / \#)\end{array}$ \\
\hline $\begin{array}{l}\text { No. of RT \# } \\
\text { Intervention }\end{array}$ & $27-28$ & $28+/-2$ & $25-30$ & $25+/-8$ & 25 & $>25$ & 25 & $15+/-5$ & $25+/-5$ & $16 / 25$ \\
\hline Topical steroid & 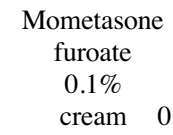 & $\begin{array}{l}\text { Methyl- } \\
\text { predni } \\
\text { solone } \\
0.1 \% \text { cream }\end{array}$ & $\begin{array}{c}\text { Beta- } \\
\text { methasone } \mathrm{r} \\
0.1 \% \\
\text { ointment } 1\end{array}$ & $\begin{array}{c}\text { Beclo- } \\
\text { methasone } \\
\text { spray } \\
100 \mu \mathrm{g} / \text { puff }\end{array}$ & $\begin{array}{c}\text { Beta- M } \\
\text { methasone } \\
0.1 \% \\
\text { ointment }\end{array}$ & $\begin{array}{l}\text { Mometasone } \\
\text { furoate } \\
0.1 \% \\
\text { cream }\end{array}$ & $\begin{array}{l}\text { Beta- } \\
\text { methasone } \\
\text { cream }\end{array}$ & $\begin{array}{l}\text { Mometasone } \\
\text { furoate } \\
0.1 \% \\
\text { cream }\end{array}$ & $\begin{array}{c}\text { Hydro- } \\
\text { cortisone } \\
1 \% \\
\text { cream }\end{array}$ & $\begin{array}{l}\text { Beta- } \\
\text { methasone } \\
\text { cream }\end{array}$ \\
\hline Comparison & $\begin{array}{l}\text { Placebo De } \\
\text { cream }\end{array}$ & $\begin{array}{l}\text { Dexpanthenol } \\
\text { cream }\end{array}$ & $\begin{array}{l}\text { Placebo } \\
\text { ointment }\end{array}$ & $\begin{array}{c}\text { No } \\
\text { treatment }\end{array}$ & $\begin{array}{l}\text { Petrolatum/ } \\
\text { No treatment }\end{array}$ & $\begin{array}{l}\text { Placebo } \\
\text { t cream }\end{array}$ & $\begin{array}{l}\text { Essex cream/ } \\
\text { canoderm cream }\end{array}$ & $\begin{array}{l}\text { Diprobase } \\
\text { cream }\end{array}$ & $\begin{array}{c}\text { Placebo } \\
\text { cream }\end{array}$ & $\begin{array}{l}\text { Essex } \\
\text { cream }\end{array}$ \\
\hline $\begin{array}{l}\text { Frequency of } \\
\text { application }\end{array}$ & $\begin{array}{l}\text { Twice weekly } \\
\text { x first } 2 \text { weeks } \\
\text { of RT, then } \\
\text { once daily }\end{array}$ & $\begin{array}{ll}\text { y } & \text { Twice } \\
\text { xs } & \text { daily }\end{array}$ & $\begin{array}{l}\text { Twice } \\
\text { daily }\end{array}$ & $\begin{array}{l}\text { Twice } \\
\text { daily }\end{array}$ & $\begin{array}{l}\text { Twice } \\
\text { daily }\end{array}$ & $\begin{array}{l}\text { Once } \\
\text { daily }\end{array}$ & $\begin{array}{l}\text { Once } \\
\text { daily }\end{array}$ & $\begin{array}{l}\text { Once } \\
\text { daily }\end{array}$ & $\begin{array}{l}\text { Twice } \\
\text { daily }\end{array}$ & $\begin{array}{l}\text { Once } \\
\text { daily }\end{array}$ \\
\hline $\begin{array}{l}\text { Duration of } \\
\text { RT treatment } \\
\text { Outcome }\end{array}$ & 6 weeks & 6 weeks & 5-6 weeks : & 5-6 weeks & 5 weeks & $>5$ weeks & 5 weeks & 3-4 weeks & 5-6 weeks & $3 / 5$ weeks \\
\hline RD scale & $\begin{array}{l}\text { Clinical } \\
\text { scale }\end{array}$ & $\begin{array}{l}\text { Clinical } \\
\text { scale }\end{array}$ & RTOG & $\begin{array}{l}\text { Clinical } \\
\text { scale }\end{array}$ & RTOG & CTCAE & RTOG & $\begin{array}{l}\text { Modified } \\
\text { RTOG }\end{array}$ & CTCAE & RTOG \\
\hline Pain/Qol scale & VAS & $\begin{array}{l}\text { Skindex-16 } \\
\text { \& SF-36 }\end{array}$ & Visual & - & $\mathrm{C}$ & $\begin{array}{l}\text { CTCAE SE } \\
\text { diary, } \\
\text { Skindex-16, }\end{array}$ & $\begin{array}{l}\text { VAS \& } \\
\text { DLQI }\end{array}$ & DLQI & $\begin{array}{l}\text { VAS \& } \\
\text { DLQI }\end{array}$ & VAS \\
\hline $\begin{array}{l}\text { Baseline } \\
\text { assessment } \\
\text { of RD }\end{array}$ & Yes & Yes & - & - & - & Yes & Yes & Yes & Yes & Yes \\
\hline $\begin{array}{l}\text { Baseline } \\
\text { assessment of } \\
\text { pain/Qol }\end{array}$ & UNCLEAR & Yes & - & - & - & Yes & - & yes & UNCLEAR & Yes \\
\hline $\begin{array}{l}\text { Intervention } \\
\text { duration }\end{array}$ & 9 weeks & 8 weeks & 6-7 weeks & 5-6 weeks & 7 weeks & $>5$ weeks & 7 weeks & 5 weeks & 6-7 weeks & $5 / 7$ weeks \\
\hline Study duration & 9 weeks & 8 weeks & 9-10 weeks & 9 weeks & 7 weeks & $>7$ weeks & 7 weeks & 6 weeks & 6-7 weeks & $4 / 6$ weeks \\
\hline Dropout & $\begin{array}{c}1 \text { (MF) } 2 \\
2 \%\end{array}$ & $\begin{array}{c}2 \text { (methyl) } \\
8.7 \%\end{array}$ & $\begin{array}{c}4 \text { (placebo) } \\
5.3 \%\end{array}$ & $0 \%$ & $\begin{array}{c}7 \\
12 \%\end{array}$ & $\begin{array}{c}10(6 \mathrm{MF} / 4 \\
\text { placebo }) \\
1.78 \%\end{array}$ & $\begin{array}{c}2 \text { (cano) } \\
1.9 \%\end{array}$ & $\begin{array}{c}6(3 \mathrm{MF} / 3 \mathrm{D}) \\
5 \%\end{array}$ & 0 & 0 \\
\hline $\begin{array}{l}\text { Eligible for } \\
\text { evaluation }\end{array}$ & $\begin{array}{c}49(24 \\
\mathrm{MF} / 25 \\
\text { placebo })\end{array}$ & $\begin{array}{l}21(10 \\
\text { methyl/ } \\
11 \mathrm{dex})\end{array}$ & $\begin{array}{c}72(38 \\
\text { beta/34 } \\
\text { placebo) }\end{array}$ & $\begin{array}{c}60(30 \\
\text { beclo/ } \\
30 \text { no trt })\end{array}$ & $\begin{array}{l}51 \text { (19 beta/ } \\
17 \text { petro/ } \\
15 \text { no trt })\end{array}$ & $\begin{array}{c}166(84 \\
\text { MF/82 } \\
\text { placebo) }\end{array}$ & $\begin{array}{c}102(53 \\
\text { Beta/ } 49 \\
\text { essex-cano) }\end{array}$ & 114 & 50 & 202 \\
\hline
\end{tabular}

BCS: Breast conservative surgery; MF: mometasone furoate; methyl: methylpredinisolone; beclo: beclomethasone; cano: canoderm cream; beta: betamethasone; dex: dexpanthenol; no trt: no treatment; petro: petrolatum; D: diprobase; hydro: hydrocortisone; VAS: visual analogue scale; CTCAE: common terminology criteria for adverse events; RTOG: radiation therapy oncology group; Qol: quality of life; RT \#: number of radiation therapy fraction; RT: radiation therapy; Gy: gray; RD: radiation dermatitis; MRM: modified radical mastectomy; SD: standard deviation; Linac: linear accelerator; MV: mega voltage; MeV: mega electron voltage; DLQI: dermatology life questionnaire index; SF-36: short form health survey. 


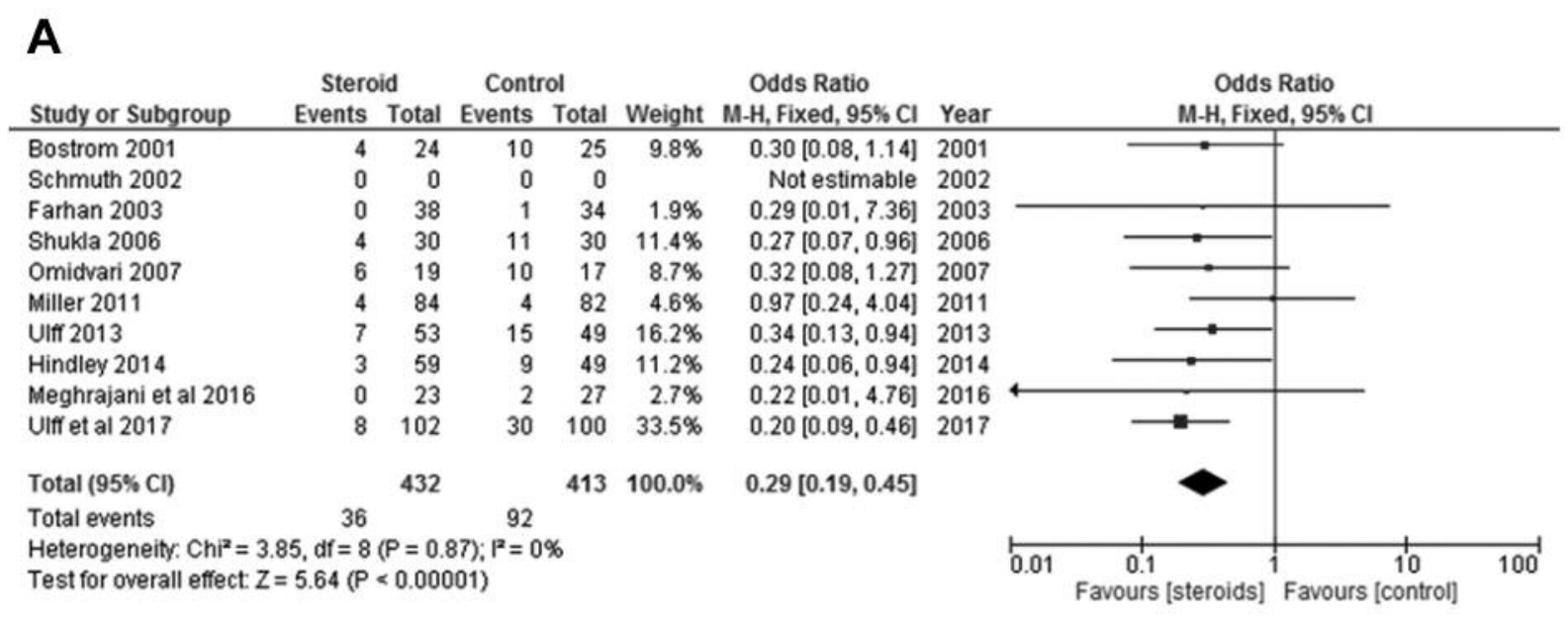

B

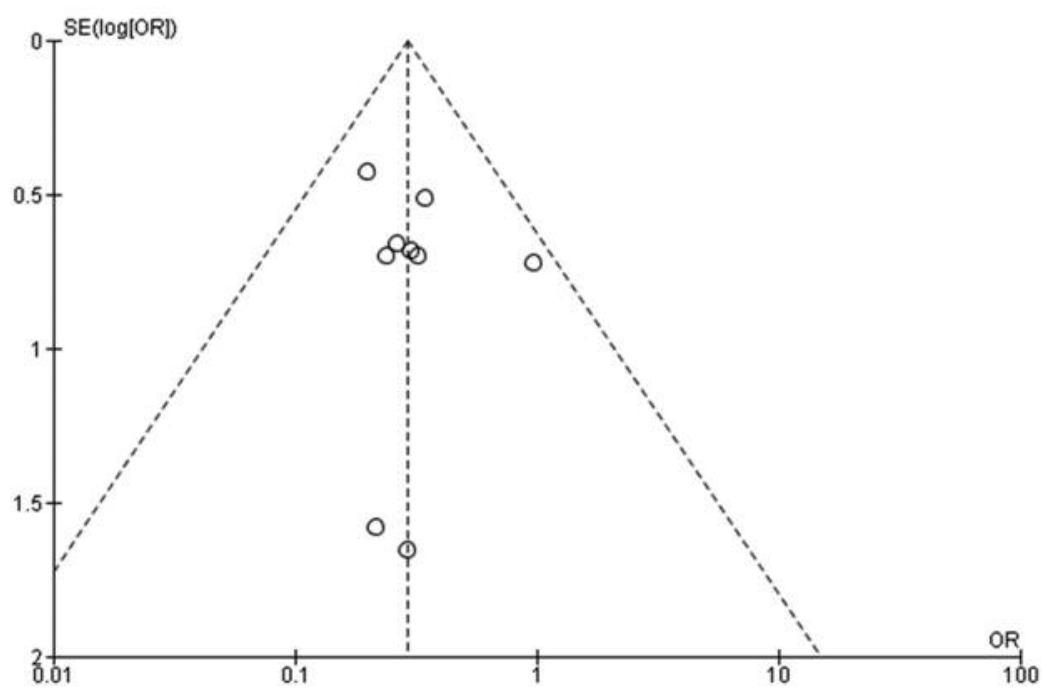

Figure 3. A) Outcome: Incidence of wet desquamation. A: Forest plot of comparison: steroid vs. control. df: degree of freedom, Fixed: Fixed Effect, MH: Mantel Haenszel. B) Outcome: Incidence of wet desquamation. B: Funnel plot of comparison: steroid vs. control. OR: Odd ratio; SE: standard error.

deviation were read from the graphs presented $(7,33)$. However, they were calculated from the tables presented in four studies $(8,27,31,32)$ and were given in two $(26,29)$. One study was excluded in this analysis because there was no data on the mean RD score (30). Raw data was requested for one study as the mean and standard deviation could not be extracted or calculated from the data provided (28). All studies showed a lower mean score in the steroid group versus the control group (Table IV). Pooling results for nine RCTs; the data consisted of 806 participants. Due to the variations in the scaled used in the studies; standardised mean difference was employed for a quantitative analysis of these results. Meta analysis's results demonstrated a reduction in mean RD scores across the trials (SMD: -0.47 , 95\% CI: $-0.61-0.33, p<0.00001)$, with evidence of heterogeneity $\left(\mathrm{Chi}^{2}=15.79 ; \mathrm{df}: 8 ; p=0.05 ; \mathrm{I}^{2}=49 \%\right)$ (Figure 4A). An associated funnel plot (Figure 4B).

Patients' subjective report of pain. Patients' subjective report of pruritus and burning was explained and measured in six trials $(8,26,28,29,31,32)$. All six studies reported less pruritus and burning in the steroid group compared to the control. Four studies showed significant results $(26,28,29$, 31) (Table IV). Pain was reported in four trials however there was no significant difference between the two arms of study in the trials $(8,26,29,31)$ (Table IV). 
Table IV. Primary and secondary outcome

\begin{tabular}{|c|c|c|c|c|c|c|c|c|c|c|}
\hline Outcome & $\begin{array}{l}\text { Bostrom } \\
\text { et al. } \\
(2001) \\
\text { MF vs. } \\
\text { placebo }\end{array}$ & $\begin{array}{l}\text { Schmuth } \\
\text { et al. } \\
\text { (2002) } \\
\text { methyl } \\
\text { vs. dex }\end{array}$ & $\begin{array}{c}\text { Farhan } \\
\text { et al. } \\
(2003) \\
\text { beta } \\
v s . \text { placebo }\end{array}$ & $\begin{array}{l}\text { Shukla } \\
\text { et al. } \\
(2006) \\
\text { beclo } \\
\text { vs. no trt }\end{array}$ & $\begin{array}{l}\text { Omidvari } \\
\text { et al. } \\
(2007) \\
\text { beta } v s . p \\
\text { etro } v s . \text { no trt }\end{array}$ & $\begin{array}{l}\text { Miller } \\
\text { et al. } \\
(2011) \\
\text { MF } v s . \\
\text { placebo }\end{array}$ & $\begin{array}{c}\text { Ulff } \\
\text { et al } \\
(2013) \\
\text { beta } v s \text {. } \\
\text { essex } v s \text {. cano }\end{array}$ & $\begin{array}{c}\text { Hindley } \\
\text { et al. } \\
(2014) \\
\text { MF } \\
\text { vs. D }\end{array}$ & $\begin{array}{l}\text { Meghrajani } \\
\text { et al. } \\
(2016) \\
\text { hydro } v s . \\
\text { placebo }\end{array}$ & $\begin{array}{l}\text { Ulff } \\
\text { et al. } \\
(2017) \\
\text { beta } v s \text {. } \\
\text { essex }\end{array}$ \\
\hline \multicolumn{11}{|l|}{ Primary outcomes } \\
\hline $\begin{array}{l}\text { Incidence of wet } \\
\text { desquamation } \\
\text { (grade 3) }\end{array}$ & $\begin{array}{l}\text { MF: } 16.67 \% \\
\text { Placebo: } \\
40 \%\end{array}$ & 6 & $\begin{array}{c}\text { Beta: } 0 \% \text { E } \\
\text { Placebo: } \\
2.9 \%\end{array}$ & $\begin{array}{c}\text { Beclo: } 13.33 \% \\
\text { No trt: } \\
36.66 \%\end{array}$ & $\begin{array}{l}\text { Beta: } 35 \% \\
\text { Petro: } 60 \% \\
\text { No trt: } 45 \%\end{array}$ & $\begin{array}{c}\text { MF: } 4.76 \% \\
\text { Placebo: } \\
4.87 \%\end{array}$ & $\begin{array}{c}\text { Beta: } 15 \% \\
\text { Essex-cano: } \\
30.6 \%\end{array}$ & $\begin{array}{l}\text { MF: } 4.8 \% \\
\text { D: } 15.5 \%\end{array}$ & $\begin{array}{c}\text { Hydro: 0\% } \\
\text { Placebo: } \\
7.41 \%\end{array}$ & $\begin{array}{l}\text { Beta: } 7.84 \% \\
\text { Essex: } 30 \%\end{array}$ \\
\hline \multicolumn{11}{|c|}{ Secondary outcomes } \\
\hline $\begin{array}{l}\text { Mean RD } \\
\text { score +SD }\end{array}$ & $\begin{array}{c}3.25 \pm 1.39 \\
v s . \\
4.32 \pm 1.49\end{array}$ & $\begin{array}{c}1.6 \pm 0.25 \\
v s . \\
2.2 \pm 0.4\end{array}$ & $\begin{array}{c}1.026 \pm 0.367 \\
v s . \\
1.429 \pm 0.558\end{array}$ & 7 & $\begin{array}{c}1.3 \pm 0.3 \\
v s . \\
1.5 \pm 0.3\end{array}$ & $\begin{array}{c}1.167 \pm 0.848 \\
v s . \\
1.341 \pm 0.805\end{array}$ & $\begin{array}{c}1.623 \pm 0.79 \\
\text { vs. } \\
2.163 \pm 0.746\end{array}$ & $\begin{array}{c}1.427 \pm 0.564 \\
v s . \\
1.664 \pm 0.588\end{array}$ & $\begin{array}{c}0.713 \pm 0.204 \\
\text { vs. } \\
0.874 \pm 0.284\end{array}$ & $\begin{array}{c}1.768 \pm 1.008 \\
v s . \\
1.97 \pm 0.846\end{array}$ \\
\hline $\begin{array}{l}\text { Pain and } \\
\text { subjective report }\end{array}$ & $\begin{array}{c}\text { No difference } \\
\text { in pain } \\
(p=0.42) . \\
\text { Less itching } \\
(p=0.069) \\
\text { and burning } \\
(p=0.087) \\
\text { in MF }\end{array}$ & se & $\begin{array}{c}\text { No difference } \\
\text { in pain. } \\
\text { Less burning } \\
\text { and pruritus } \\
\text { in beta } v s . \\
\text { placebo, } \\
p<0.05\end{array}$ & e & - & $\begin{array}{l}\text { MF: } 2.5 \text { vs. } \\
\text { Placebo: } \\
2.9(p=0.15) \\
\text { Less } \\
\text { itching and } \\
\text { burning } \\
(p=0.002, \\
0.02)\end{array}$ & $\begin{array}{l}\text { Less in beta, } \\
p=0.048- \\
\text { (burning, } \\
\text { itching, } \\
\text { irritation) }\end{array}$ & - & $\begin{array}{c}\text { No } \\
\text { difference } \\
\text { in pain. } \\
\text { Less } \\
\text { pruritus } \\
\text { in hydro } \\
v s . \text { placebo } \\
(p=0.032)\end{array}$ & $\begin{array}{l}\text { Less } \\
\text { itching, } \\
\text { burning } \\
\text { and } \\
\text { irritation } \\
\text { in beta } \\
v s \text {. essex }\end{array}$ \\
\hline Qol report & $\begin{array}{l}1 \\
1 \\
7\end{array}$ & $\begin{array}{c}\text { Methyl: } 4 \\
\text { out of } 7 \\
\text { aspects } \\
\text { worsened } \\
v s . \text { dex: } \\
7 \text { out of } 7\end{array}$ & 7 & - & - & $\begin{array}{c}\text { No } \\
\text { diference }\end{array}$ & $\begin{array}{c}\text { No } \\
\text { difference } \\
\text { in DLQI }\end{array}$ & $\begin{array}{l}\text { Less } \\
\text { DLQI } \\
\text { in MF }\end{array}$ & $\begin{array}{c}\text { Lower } \\
\text { DLQI score } \\
\text { in hydro } \\
(2.22 v s . \\
2.48) . \\
p=0.069\end{array}$ & - \\
\hline
\end{tabular}

RD: Radiation dermatitis; SD: standard deviation; vs: versus; methyl: methyl predinisolone; dex: dexpanthenol; MF: mometasone furoate; beta: betamethasone; DLQI: dermatology life questionnaire index; beclo: beclometasone; No trt: no treatment; petro: petrolatum; cano: canoderm cream; D: diprobase; hydro: hydrocortisone.

Quality of life. Five trials assessed Qol in their participants (7, 26-29). Skindex-16 (7, 28) and DLQI (27-29) were the tools employed to examine skin related Qol. Schmuth et al. observed that there was worsening in all aspects of Qol in the dexpanthenol arm compared to four out of seven aspects in the methylprednisolone arm (7) (Table IV). There was no difference in Qol between the groups in two studies Ulff et $a l$, and Miller et al. however Hindley et al. and Meghrajani et al. found less DLQI scores in their steroid group compared to the control group (26-29) (Table IV). None of the studies reported a significant difference between the arms of study with regards to Qol except in Hindley et al., however the $p$-value was not stated (27).

\section{Discussion}

Radiation dermatitis is one of the most common side-effects of radiotherapy and varies in grade from mild erythema to more severe reactions of wet desquamation; ulceration and sometimes necrosis (6). Wet desquamation mainly occurs in dose of excess of 20 Gy $(4,34)$. Many factors have been reported to influence the severity of RD in patients (35), patients' related factors (e.g. smoking, bra size) and treatment factors (e.g. beam energy, dose, treatment techniques, chemotherapy, tamoxifen) $(8,21,36)$. IMRT significantly reduced the severity of RD in breast cancer patients compared to conventional techniques $p<0.001$, but it is not the standard of care in breast cancer radiotherapy (37). Smaller bra size was found to be associated with decreased risk of wet desquamation in a multivariate analysis $(38,39)$.

Despite the vast improvement in radiation technology, RD remains a concern $(6,39,40)$. Reduced skin sparing effect because of the commonly used lower beam energy in breast cancer radiotherapy coupled with tangential beam arrangement mean that a lot of patients will experience some form of $\mathrm{RD}(40,41)$. Consensus regarding care for $\mathrm{RD}$ is still lacking and there is an urgent need for its appropriate management to improve outcome for this patient cohort (6).

Efforts have been made to quantify the degree of skin reaction and this is seen in the development of the RTOG and CTCAE scales for skin reaction; however more 


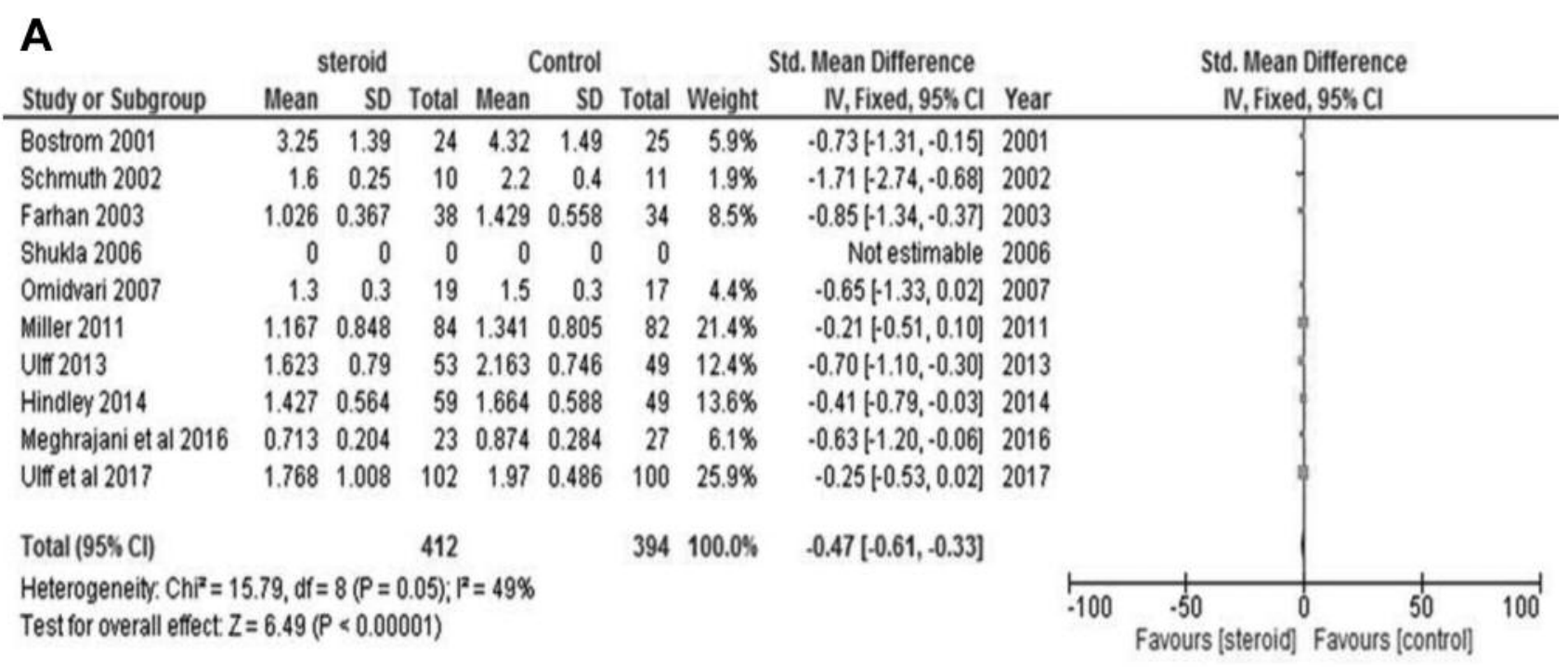

B

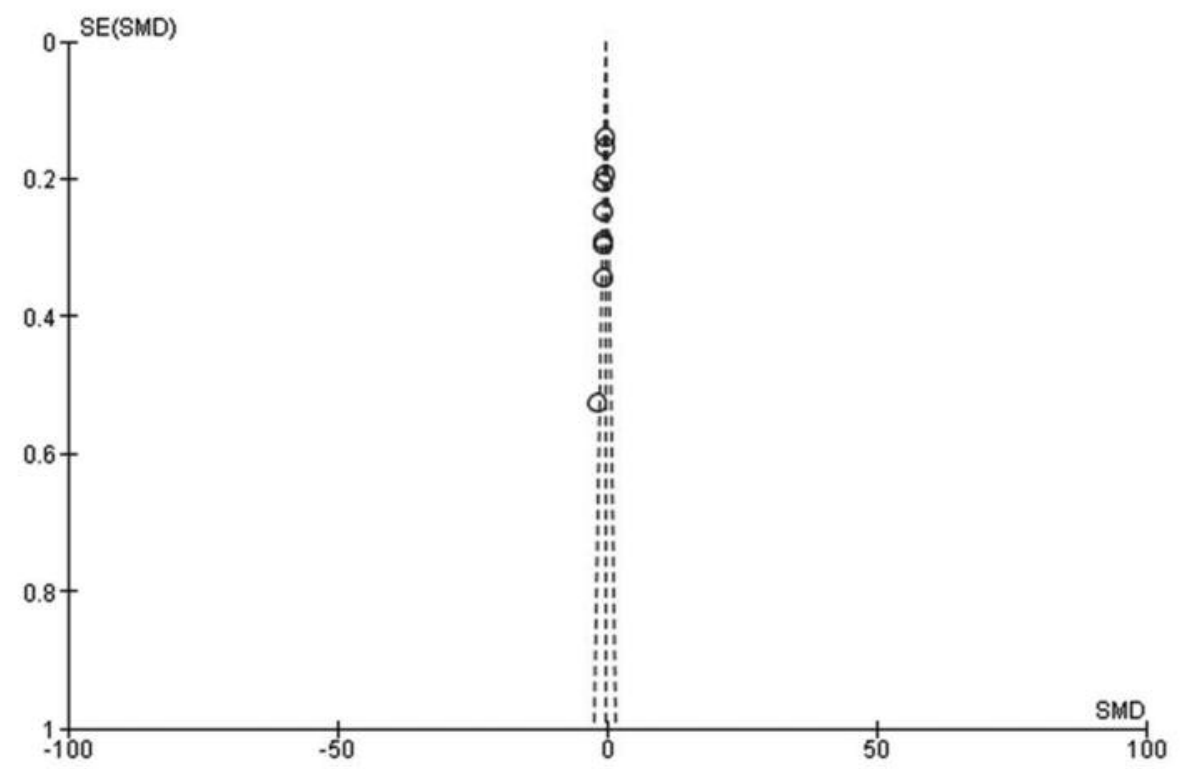

Figure 4. A) Outcome: Mean acute radiation dermatitis. A: Forest plot of comparison: steroid vs. control.df: Degree of freedom, Fixed: fixed effect, IV: inverse variance, std: standard. B) Outcome: Mean acute radiation dermatitis. B: Funnel plot of comparison: steroid vs. control. SMD: Standard mean difference, SE: standard error.

research is needed to validate these tools for accurate and precise assessment (42). Also grade 3 RTOG and CTCAE $\mathrm{RD}$ has been chosen as the endpoint of this review as it is the least ambiguous description of wet desquamation according to these scales. Particularly of interest are the clinical scales developed by authors used in some of the trials included in this review $(7,8,30)$. These scales were not validated nor previously published and thus could be a potential source of bias.
Non-steroidal agents have been tested in trials to determine their impact on RD but studies have failed to show a positive effect (42). Steroids are already in practice and are now emerging as a suitable alternative $(6,15,22)$. Clinical evidence to date is insufficient to demonstrate their clinical benefit. Therefore, it was necessary to pool the results of these trials together in a systematic review to establish a grounded and more concrete conclusion that could in turn inform practice. The topical corticosteroids in this review 
vary from mild to potent and the vehicles used were mainly cream and ointment except in one study where aerosol was employed. The reason for this was to avoid the bolus effect however the increase in surface dose is not significant when a thin layer $(<2 \mathrm{~mm})$ of product is applied (43). The maximum application of treatment in the studies included in this review did not exceed twice daily (Table III).

A previous review by Meghrajani et al. identified that the incidence of wet desquamation was 2.5-times less likely with the use of topical steroids (44). The meta-analysis included 383 patients. Our results had 845 patients and demonstrated that the risk is at least 5 times less likely. This is a marked improvement however it must be stated that the qualities of the trials included could have influenced the outcome observed. In order to reduce bias towards steroids it was necessary to examine the trials individually and try to make meaning of their results. It is expected that in trials where superficial X-rays and Cobalt-60 were employed for treatment, an increased incidence of wet desquamation would be reported $(30,31,33)$. This is due to a reduced skin sparing effect compared to the other trials where at least $5 \mathrm{MV}$ were used (40). Also, the trials with the higher beam energies had much larger sample sizes compared to the former trials $(7,8$, 26-28). Surprisingly Farhan et al. had no event in the steroid arm and only one incidence of wet desquamation in the control arm despite having employed Cobalt-60 as the method of radiation therapy treatment delivery (31). The $95 \%$ CI (0.01-7.38) for this trial was quite wide, implying a degree of uncertainty in the effect size and necessitating caution when interpreting the results. Upon assessing the internal validity of this trial incomplete outcome data and selective reporting were identified. Nonetheless our result agrees with the systematic review previously done by Salvo et al. reporting that topical corticosteroid agents significantly reduce the severity of $\mathrm{RD}(6)$.

The mean RD score was lower in the steroid group than in the control arm. This showed that steroid is effective in reducing the grade of $\mathrm{RD}$ observed in patients and that majority of patients would not be experiencing the more severe effects. Our result is on par with that of Meghrajani et al. (SMD: $-0.47,95 \% \mathrm{CI}:-0.61-0.33, p<0.00001$ ) even though more participants were included in our pooled analysis (345 vs. 806) (44). Heterogeneity was present $\left(\mathrm{I}^{2}=49 \%\right)$ but less than in Meghrajani et al. It was sometimes difficult to extract data from the graphs provided by some of the authors and in some the raw data was absent. This could have resulted in an overestimation of effect and the heterogeneity observed in our review.

The signs and symptoms of RD are often expressed by patients as pain, burning, itching (45). Six trials examined itching and burning $(8,26,28,29,31,32)$. Tools employed included the Visual Analogue Scale. There was less symptom of burning and itching in the steroid group compared to the control group. This was somewhat expected as standard management of pruritus associated with inflammatory diseases include topical corticosteroids such as mometasone and betamethasone $(46,47)$. Five of the trials had either mometasone or betamethasone as intervention. Therefore it was not unusual that fewer symptoms were observed in the steroid arm. In the systematic review by Koukourakis et al, it was suggested that a low dose corticosteroid may be beneficial in reducing itching and irritation (48). This was also recommended in the guideline by Wong et al. (49). For pruritus and burning, the results of three trials out of six were significant at week $5, p<0.05,0.002,0.032(26,29,31)$ (Table IV). This however contrasted with the result of the review by Bolderston et al. where no significant difference was detected between the steroid arm and the control (18). Although the study by Ulff et al. observed significant difference $(p=0.048)$ between the groups at week 6 , the time endpoint for this review was week 5 (28).

Pain was assessed in four trials $(8,26,29,31)$. Baseline measurement of pain was performed (26); it was unclear (8, 29) and was not performed (31). There was no significant difference between the steroid arm and the control arm, leaving us to conclude that topical corticosteroid does not have any impact on pain associated with RD in line with the results of Bolderston et al. (18).

The distress and unfavourable impact of RD on quality of life is also an important area of assessment $(21,40,50,51)$. Radiation dermatitis can have a significant effect on patients' Qol and can determine the prescribed therapeutic radiation dose $(6,40,49)$. Only five of the ten included trials examined the impact of RD on participants' Qol, highlighting the need for more emphasis to be placed on research in this area $(7,26-29)$ (Table IV). All five trials used either Skindex-16 or DLQI in assessing Qol. These tools have been validated in dermatology as standard assessment tools which in turn made their results plausible (52). Baseline assessment of Qol was conducted in three trials (7, $26,27)$ however it was unclear in two $(28,29)$. Although heterogeneity in the method of reporting made quantitative analysis unfeasible, the use of topical steroid improved Qol in patients however the significance of this result could not be established. Hindley at al stated that mometasone furoate significantly improved Qol but the $p$-value wasn't given and thus we were unable to make a definitive recommendation.

Limitations. Even though all of the RCTs were deemed to be of reasonable quality, a few high-risk features including incomplete outcome data reporting and unclear domains could have impacted on the outcome. The included studies used different tools in measuring RD leading to differences in outcome reporting. In some studies raw data was not available and was sometimes difficult to extract from studies therefore we were unable to include all studies in meta-analysis. 
Although the impact of this was somewhat minimised by contacting authors for raw data and clarification in their results. We were unable to quantitatively analyze the impact of corticosteroids on subjective symptoms and Qol to establish its significance due to heterogeneity in reporting methods.

\section{Conclusion}

This review identified that topical corticosteroids can be effective in reducing the incidence of wet desquamation and lessen the mean RD scores. Administration of these agents can also improve Qol and lower subjective symptoms. Future studies are required to validate tools needed to evaluate $\mathrm{RD}$, assess the comparative effectiveness of topical steroids, and address the potential adverse effects not examined in this review.

\section{References}

1 Hymes SR, Strom EA and Fife C: Radiation dermatitis: Clinical presentation, pathophysiology, and treatment 2006. J Am Acad Dermatol 54(1): 28-46, 2006.

2 Maddocks-Jennings W, M Wilkinson J and Shillington D: Novel approaches to radiotherapy-induced skin reactions: A literature review. Complement Ther Clin Pract 11(4): 224-231, 2005.

3 Wolbarst AB, Wiley AL Jr., Nemhauser JB, Christensen DM and Hendee WR: Medical response to a major radiologic emergency: a primer for medical and public health practitioners. Radiology 254(3): 660-677, 2010.

4 Mendelsohn FA, Divino CM, Reis ED and Kerstein MD: Wound care after radiation therapy. Adv Skin Wound Care 15(5): 216$224,2002$.

5 Sekiguchi K, Ogita M, Akahane K, Haga C, Ito R, Arai S, Ishida $\mathrm{Y}$, Tsukada $\mathrm{Y}$ and Kawamori J: Randomized, prospective assessment of moisturizer efficacy for the treatment of radiation dermatitis following radiotherapy after breast-conserving surgery. Jpn J Clin Oncol 45(12): 1146-1153, 2015.

6 Salvo N, Barnes E, van Draanen J, Stacey E, Mitera G, Breen D, Giotis A, Czarnota G, Pang $\mathrm{J}$ and De Angelis C: Prophylaxis and management of acute radiation-induced skin reactions: A systematic review of the literature. Curr Oncol 17(4): 94-112, 2010.

7 Schmuth M, Wimmer MA, Hofer S, Sztankay A, Weinlich G, Linder DM, Elias PM, Fritsch PO and Fritsch E: Topical corticosteroid therapy for acute radiation dermatitis: A prospective, randomized, double-blind study. $\mathrm{Br} \mathrm{J}$ Dermatol 146(6): 983-991, 2002.

8 Bostrom A, Lindman H, Swartling C, Berne B and Bergh J: Potent corticosteroid cream (mometasone furoate) significantly reduces acute radiation dermatitis: Results from a double-blind, randomized study. Radiother Oncol 59(3): 257-265, 2001.

9 Chan RJ, Keller J, Cheuk R, Blades R, Tripcony L and Keogh $\mathrm{S}$ : A double-blind randomised controlled trial of a natural oilbased emulsion (moogoo udder cream ${ }^{\circledR}$ ) containing allantoin versus aqueous cream for managing radiation-induced skin reactions in patients with cancer. Radiat Oncol 7(121): 121, 2012.
10 Wickline M: Prevention and treatment of acute radiation dermatitis: A literature review. Oncol Nurs Forum 31(2): 237247, 2004.

11 Di Franco R, Sammarco E, Calvanese MG, De Natale F, Falivene S, Di Lecce A, Giugliano FM, Murino P, Manzo R, Cappabianca S, Muto P and Ravo V: Preventing the acute skin side effects in patients treated with radiotherapy for breast cancer: The use of corneometry in order to evaluate the protective effect of moisturizing creams. Radiat Oncol 8: 57-57, 2013.

12 Heggie S, Bryant GP, Tripcony L, Keller J, Rose P, Glendenning $\mathrm{M}$ and Heath J: A phase iii study on the efficacy of topical aloe vera gel on irradiated breast tissue. Cancer Nurs 25(6): 442-451, 2002.

13 Richardson J, Smith JE, McIntyre M, Thomas R and Pilkington $\mathrm{K}$ : Aloe vera for preventing radiation-induced skin reactions: A systematic literature review. Clin Oncol (R Coll Radiol) 17(6): 478-484, 2005.

14 Sharp L, Finnilä K, Johansson H, Abrahamsson M, Hatschek T and Bergenmar M: No differences between calendula cream and aqueous cream in the prevention of acute radiation skin reactions - results from a randomised blinded trial. Eur J Oncol Nurs 17(4): 429-435, 2013.

15 Glees JP, Mameghan-Zadeh H and Sparkes CG: Effectiveness of topical steroids in the control of radiation dermatitis: A randomised trial using $1 \%$ hydrocortisone cream and $0.05 \%$ clobetasone butyrate (eumovate). Clin Radiol 30(4): 397-403, 1979.

16 Chen MF, Chen WC, Lai CH, Hung $\mathrm{CH}$, Liu $\mathrm{KC}$ and Cheng $\mathrm{YH}$ : Predictive factors of radiation-induced skin toxicity in breast cancer patients. BMC Cancer 10: 508, 2010.

17 Pitzalis C, Pipitone N, Bajocchi G, Hall M, Goulding N, Lee A, Kingsley G, Lanchbury J and Panayi G: Corticosteroids inhibit lymphocyte binding to endothelium and intercellular adhesion: An additional mechanism for their anti-inflammatory and immunosuppressive effect. J Immunol 158(10): 5007-5016, 1997.

18 Bolderston A, Lloyd NS, Wong RK, Holden L and RobbBlenderman L: The prevention and management of acute skin reactions related to radiation therapy: A systematic review and practice guideline. Support Care Cancer 14: 802, 2006.

19 Aistars J: The validity of skin care protocols followed by women with breast cancer receiving external radiation. Clin J Oncol Nurs 10(4): 487-492, 2006.

20 Naylor W and Mallett J: Management of acute radiotherapy induced skin reactions: A literature review. Eur J Oncol Nurs 5(4): 221-233, 2001.

21 Feight D, Baney T, Bruce S and McQuestion M: Putting evidence into practice. Clin J Oncol Nurs 15(5): 481-492, 2011.

22 Berkey FJ: Managing the adverse effects of radiation therapy. Am Fam Physician 82(4): 381-388, 394, 2010.

23 Moher D, Liberati A, Tetzlaff J and Altman DG: Preferred reporting items for systematic reviews and meta-analyses: The prisma statement. PLoS Med 6(7): e1000097, 2009.

24 Jadad AR, Moore RA, Carroll D, Jenkinson C, Reynolds DJM, Gavaghan DJ and McQuay HJ: Assessing the quality of reports of randomized clinical trials: Is blinding necessary? Control Clin Trials 17(1): 1-12, 1996.

25 Higgins JPT, Altman DG, Gøtzsche PC, Jüni P, Moher D, Oxman AD, Savović J, Schulz KF, Weeks L and Sterne JAC: 
The cochrane collaboration's tool for assessing risk of bias in randomised trials. BMJ 343: d5928, 2011.

26 Miller RC, Schwartz DJ, Sloan JA, Griffin PC, Deming RL, Anders JC, Stoffel TJ, Haselow RE, Schaefer PL, Bearden JD, 3rd, Atherton PJ, Loprinzi CL and Martenson JA: Mometasone furoate effect on acute skin toxicity in breast cancer patients receiving radiotherapy: A phase iii double-blind, randomized trial from the north central cancer treatment group n06 4 . Int J Radiat Oncol Biol Phys 79(5): 1460-1466, 2011.

27 Hindley A, Zain Z, Wood L, Whitehead A, Sanneh A, Barber D and Hornsby R: Mometasone furoate cream reduces acute radiation dermatitis in patients receiving breast radiation therapy: Results of a randomized trial. Int J Radiat Oncol Biol Phys 90(4): 748-755, 2014.

28 Ulff E, Maroti M, Serup J and Falkmer U: A potent steroid cream is superior to emollients in reducing acute radiation dermatitis in breast cancer patients treated with adjuvant radiotherapy. A randomised study of betamethasone versus two moisturizing creams. Radiother Oncol 108(2): 287-292, 2013.

29 Meghrajani CF, Co HS, Arcillas JG, Maano CC and Cupino NA: A randomized, double-blind trial on the use of $1 \%$ hydrocortisone cream for the prevention of acute radiation dermatitis. Expert Rev Clin Pharmacol 9(3): 483-491, 2016.

30 Shukla PN, Gairola M, Mohanti BK and Rath GK: Prophylactic beclomethasone spray to the skin during postoperative radiotherapy of carcinoma breast: A prospective randomized study. Indian J Cancer 43(4): 180-184, 2006.

31 Farhan F, Kazemian A and Alagheband H: Topical betamethasone for the prevention of acute radiation dermatitis in breast cancer patients. Iran J Radiat Res 1: 105-111, 2003.

32 Ulff E, Maroti M, Serup J, Nilsson M and Falkmer U: Prophylactic treatment with a potent corticosteroid cream ameliorates radiodermatitis, independent of radiation schedule: A randomized double blinded study. Radiother Oncol 122(1): 5053, 2017.

33 Omidvari S, Saboori H, Mohammadianpanah M, Mosalaei A, Ahmadloo N, Mosleh-Shirazi MA, Jowkar F and Namaz S: Topical betamethasone for prevention of radiation dermatitis. Indian J Dermatol Venereol Leprol 73(3): 209, 2007.

34 Bey E, Prat M, Duhamel P, Benderitter M, Brachet M, Trompier F, Battaglini P, Ernou I, Boutin L, Gourven M, Tissedre F, Crea $\mathrm{S}$, Mansour CA, de Revel T, Carsin H, Gourmelon P and Lataillade JJ: Emerging therapy for improving wound repair of severe radiation burns using local bone marrow-derived stem cell administrations. Wound Repair Regen 18(1): 50-58, 2010.

35 Ryan JL: Ionizing radiation: The good, the bad, and the ugly. J Invest Dermatol 132: 985-993, 2012.

36 Sharp L, Johansson H, Hatschek T and Bergenmar M: Smoking as an independent risk factor for severe skin reactions due to adjuvant radiotherapy for breast cancer. Breast 22(5): 634-638, 2013.

37 Freedman GM, Anderson PR, Li J, Eisenberg DF, Hanlon AL, Wang L and Nicolaou N: Intensity modulated radiation therapy (imrt) decreases acute skin toxicity for women receiving radiation for breast cancer. Am J Clin Oncol 29(1): 66-70, 2006.

38 Freedman GM, Li T, Nicolaou N, Chen Y, Ma CC and Anderson PR: Breast intensity-modulated radiation therapy reduces time spent with acute dermatitis for women of all breast sizes during radiation. Int J Radiat Oncol Biol Phys 74(3): 689-694, 2009.
39 Pignol JP, Olivotto I, Rakovitch E, Gardner S, Sixel K, Beckham W, Vu TT, Truong P, Ackerman I and Paszat L: A multicenter randomized trial of breast intensity-modulated radiation therapy to reduce acute radiation dermatitis. J Clin Oncol 26(13): 20852092, 2008.

$40 \mathrm{McQuestion} \mathrm{M}$ : Evidence-based skin care management in radiation therapy. Seminars in Oncology Nursing 22(3): 163173, 2006.

41 Fernandez-Castro M and Martin-Gil B: Efectiveness of topical therapies in patients with breast cancer that experience radiodermatitis. A systematic review. Enferm Clin 25(6): 327-343, 2015.

42 Sharp L, Johansson H, Landin Y, Moegelin IM and Bergenmar M: Frequency and severity of skin reactions in patients with breast cancer undergoing adjuvant radiotherapy, the usefulness of two assessment instruments - a pilot study. Eur J Cancer 47(18): 2665-2672, 2011.

43 Burch SE, Parker SA, Vann AM and Arazie JC: Measurement of 6-mv x-ray surface dose when topical agents are applied prior to external beam irradiation. Int J Radiat Oncol Biol Phys 38(2): 447-451, 1997.

44 Meghrajani CF, Co HCS, Ang-Tiu CMU and Roa FC: Topical corticosteroid therapy for the prevention of acute radiation dermatitis: A systematic review of randomized controlled trials. Exp Rev Clin Pharmacol 6(6): 641-649, 2013.

45 Chan RJ, Webster J, Chung B, Marquart L, Ahmed M and Garantziotis S: Prevention and treatment of acute radiationinduced skin reactions: A systematic review and meta-analysis of randomized controlled trials. BMC Cancer 14: 53, 2014

46 Patel $\mathrm{T}$ and Yosipovitch G: Therapy of pruritus. Expert Opin Pharmacother 11(10): 1673-1682, 2010.

47 Elmariah SB and Lerner EA: Topical therapies for pruritus. Semin Cutan Med Surg 30(2): 118-126, 2011.

48 Koukourakis GV, Kelekis N, Kouvaris J, Beli IK and Kouloulias VE: Therapeutics interventions with anti-inflammatory creams in post radiation acute skin reactions: A systematic review of most important clinical trials. Recent Pat Inflamm Allergy Drug Discov 4(2): 149-158, 2010.

49 Wong RKS, Bensadoun RJ, Boers-Doets CB, Bryce J, Chan A, Epstein JB, Eaby-Sandy B and Lacouture ME: Clinical practice guidelines for the prevention and treatment of acute and late radiation reactions from the mascc skin toxicity study group. Supportive Care in Cancer 21(10): 2933-2948, 2013.

50 Harper JL, Franklin LE, Jenrette JM and Aguero EG: Skin toxicity during breast irradiation: Pathophysiology and management. South Med J 97(10): 989-993, 2004.

51 Schnur JB, Love B, Scheckner BL, Green S, Wernicke AG and Montgomery GH: A systematic review of patient-rated measures of radiodermatitis in breast cancer radiotherapy. Am J Clin Oncol 34(5): 529-536, 2011.

52 Fernandez-Penas $\mathrm{P}$, Jones-Caballero $\mathrm{M}$, Espallardo $\mathrm{O}$ and Garcia-Diez A: Comparison of skindex-29, dermatology life quality index, psoriasis disability index and medical outcome study short form 36 in patients with mild to severe psoriasis. $\mathrm{Br}$ J Dermatol 166(4): 884-887, 2012.

Received July 6, 2017

Revised August 15, 2017 Accepted August 31, 2017 